\title{
Una aproximación a la noción relación con el saber, revisada a la luz de la teoría del sujeto de Lacan, para aplicar en la investigación de los procesos productivos de arte visual en estudiantes universitarios
}

\author{
An approach to the notion of relation to knowledge, revised in the light of \\ Lacan's theory of the subject, to be applied in the investigation of the \\ production processes of visual art in university students
}

Magali Pastorino $^{1}$

\begin{abstract}
RESUMEN
La noción relación con el saber tiene vigencia en el campo de la investigación en educación e introduce, dada su densidad conceptual, la cuestión del sujeto y el saber.

En la vía de Beillerot, ${ }^{2}$ dicha noción entraña un proceso creador singular, pertinente para la enseñanza artística universitaria en arte visual, por lo que, profundizar en ella, actualiza el campo de estudios de los procesos productivos.

No obstante, su origen radica en un sintagma de la obra lacaniana de los años sesenta. A la luz de dicha obra, nos proponemos revisar los alcances de la relación con el saber para aplicar en la investigación de los procesos productivos de estudiantes avanzados universitarios de arte visual.

De esta manera, integramos las formaciones inconscientes al campo de la enseñanza artística universitaria en arte visual.
\end{abstract}

Palabras clave: enseñanza artística universitaria, subjetivación, relación con el saber, procesos productivos.

\begin{abstract}
The notion of relationship with knowledge is valid in the field of research in education and as a dense notion it places us in the question of the subject and knowledge.

In the way of Beillerot, ${ }^{3}$ this notion involves a unique creative process, which is why it is relevant for university artistic teaching in visual art and its deepening updates the field of studies of productive processes.

However, the notion originates from a phrase in Lacanian work from the 1960s.

In the light of this work, we propose to shed light on the scope of the notion to apply in the investigation of the productive processes of advanced university students of visual art.

In this way we integrate the unconscious formations to the field of university artistic teaching in visual art.
\end{abstract}

Key Words: university artistic teaching, subjectivation, relationship with knowledge, productive processes.

${ }^{1}$ Universidad de la República (Udelar). Montevideo, Uruguay. Magíster en Psicología y Educación, Facultad de Psicología, Profesora Adjunta del Departamento de las Estéticas, Instituto Escuela Nacional de Bellas Artes, Facultad de Artes, Udelar. Correo electrónico: magalipastorino@gmail.com ORCID ID: https://orcid.org/0000-0003-2258-7423

DOI: $10.15517 /$ WL.V1711.49674

Recepción: 15/10/2021 Aceptación: 30/10/2021

2 Jacky Beillerot, «La relación con el saber: una noción en formación». En Saber y relación con el saber, editado por Jacky Beillerot, Claudine Blanchard-Laville y Nicole Mosconi (Barcelona: Paidós, 1998), 4378. Y Jacky Beillerot, «Le rapport au savoir». En Formes et formations du rapport au savoir, editado por Jacky Beillerot, Claudine Blanchard-Laville y Nicole Mosconi (Paris: L'Harmattan, 2000), 39-58.

${ }^{3}$ Beillerot, «La relación con el saber: una noción en formación». Y Beillerot, «Le rapport au savoir». 


\section{Introducción}

En el marco del Doctorado en Educación (Universidad de la República, Uruguay) nos propusimos indagar cómo se relaciona la técnica artística con la subjetivación en los procesos productivos de estudiantes universitarios avanzados — de las carreras de arte visual del Instituto Escuela Nacional de Bellas Artes, en el año lectivo 2019-, y así problematizar el lugar de la técnica en su relación con el saber. Para ello, realizamos una exploración y seguimiento, en el ámbito del taller, de los procesos generados por doce estudiantes, apoyándonos en entrevistas en profundidad, anotaciones de campo y registro fotográfico. Fue así que nos encontramos con ciertas situaciones de desconexión y abandono de la producción, por parte del estudiante, las cuales surgían sin motivos aparentes. En particular, observamos procesos productivos vividos de modo satisfactorio en el comienzo que inesperadamente fueron suspendidos. Si bien estas situaciones son habituales en la actividad productiva estudiantil, cobraron mayor relevancia en el marco de nuestra investigación. Reconocerlas como fenómenos a estudiar y comprenderlas en profundidad y en su singularidad, es decir, no interpretarlas simplemente como un componente del aprendizaje, un error de planificación o un retroceso eventual, requería de una teoría del sujeto capaz de integrar la subjetivación y el deseo en el campo educativo. Es así que la noción relación con el saber nos proporcionó un nuevo punto de vista en el análisis de dichas situaciones.

Esta noción proviene del campo de la investigación francesa en educación, la cual se constituye en los años ochenta del siglo pasado. Fue definida y aplicada por tres equipos de investigadores en función de sus objetos, líneas y enfoques de investigación, cubriendo los distintos niveles de enseñanza. La línea del Centre de Recherche Education et Formation, de la Universidad París X (CREF), liderado por Jacky Beillerot, se centró en los estudios clínicos psicoanalíticos; la de Éducation, socialisation et collectivités locales, de la Universidad París VIII (ESCOL), dirigido por Bernard Charlot, se enfocó en el plano socio-antropológico del aprendizaje; y la del Institut de Recherche sur l'Enseignement des Mathématiques de Marsella (IREM), por Yves Chevallard, trató específicamente el campo didáctico de las matemáticas. 
En la actualidad, dicha noción ha sido retomada, revisada y actualizada por investigadores de países franco, hispano y luso-parlantes, ${ }^{4}$ quienes defendieron su utilidad y vigencia, aunque no hemos encontrado investigaciones concretas en el ámbito universitario de la formación productiva en arte visual.

Si bien Charlot, ${ }^{5}$ desde una perspectiva socio-antropológica, se centró en la relación del sujeto con el mundo, como un sentido socialmente construido con componentes inconscientes, y Beillerot, ${ }^{6}$ desde una perspectiva clínica, se enfocó en el deseo involucrado en el proceso creador y las apropiaciones de saber y no saber, apuntando a los procesos primarios ocurridos en el seno de la familia, ambos investigadores señalaron que la noción entraña una compleja problemática teóricaepistemológica que abarca la conceptualización del sujeto, el saber y la relación. ${ }^{7}$

Asimismo, Beillerot, ${ }^{8}$ a fines del siglo pasado, señaló la amplitud y, por ende, la imprecisión de dicha noción que, en cierta medida, es lo que le permitiría cubrir fenómenos complejos que involucran la disposición psíquica en situación, el encuentro con otros y la dimensión educacional institucional. También señaló que el efecto de objetivación, ${ }^{9}$ a nivel metodológico, proporciona una distancia óptima para relevar lo que se encuentra naturalizado en la experiencia cotidiana.

Actualmente, con respecto al estatuto epistemológico del término, Cavalcanti, ${ }^{10}$ en procura de distinguir si se trata de un concepto operatorio, una noción teórica, una teoría o una mera expresión aplicada libremente, señalaba que fue a partir del año 2000 que el estatuto de noción se consolidó por la existencia de distintas aproximaciones y contribuciones disciplinares que generaron a partir de su uso teórico diversos programas de investigación.

\footnotetext{
${ }^{4}$ José Cavalcanti, «Le rapport au savoir: émergence, propagation et institutionnalisation en tant que notion dans les domaines des Sciences de l'Éducation et des Didactiques», Academia, 19 (2020). Y Soledad Vercellino, «La relación con el saber: revisitando los comienzos del concepto». En Aprendizajes: la escuela y los (des)encuentros con el saber, comp. Soledad Vercellino (Buenos Aires: Universidad Nacional de Río Negro, 2018).

${ }^{5}$ Bernard Charlot, La relación con el saber: elementos para una teoría (Montevideo: Trilce, 2006).

${ }^{6}$ Beillerot, «La relación con el saber: una noción en formación».

${ }^{7}$ Soledad Vercellino, «Revisión bibliográfica sobre la relación con el saber: desplazamientos teóricos y posibilidades para el análisis psicopedagógico de los procesos escolares», Revista Electrónica Educare 19, n. ${ }^{\circ}$ (2015): 53-82, ISSN 1409-4258.

${ }^{8}$ Beillerot, «La relación con el saber: una noción en formación».

${ }^{9}$ Beillerot, «La relación con el saber: una noción en formación».

${ }^{10}$ Cavalcanti, «Le rapport au savoir: émergence, propagation et institutionnalisation en tant que notion dans les domaines des Sciences de l'Éducation et des Didactiques».
} 
En nuestro caso, tomamos la conceptualización proporcionada por Beillerot, ${ }^{11}$ ya que, operativamente, nos permitió organizar los interrogantes de la investigación y enmarcar teóricamente las situaciones observadas para profundizar en los procesos productivos estudiantiles, en virtud a una subjetivación referida al obrar artístico. ${ }^{12}$

También revisamos la conceptualización de sujeto y saber que subyace en el sintagma lacaniano para comprender sus alcances epistémicos y su densidad teórica. Pues tanto Beillerot como Charlot ${ }^{13}$ afirmaron que su procedencia se encuentra en un sintagma utilizado por Lacan en los años sesenta, algunos investigadores, como Cavalcanti y Vercellino, ${ }^{14}$ lo confirmaron en sus trabajos.

En efecto, el sintagma se ubica en dos comunicaciones realizadas por Lacan: la que presentó en Royaumont, que se titula «Subversión del sujeto y dialéctica del deseo en el inconsciente freudiano», en $1960,{ }^{15}$ y la lección de apertura del seminario sobre el objeto del psicoanálisis en l'École Normale Supérieure, entre 1965 y 1966, publicada como «La ciencia y la verdad». ${ }^{16}$

En pocas palabras, Lacan desarrolla la teoría de sujeto del significante, con base en dicho sintagma, y en este marco nos sugiere una subjetivación por el significante.

Al revisar el sintagma en los textos lacanianos, nos topamos con la aventura intelectual $^{17}$ que nos legó la teoría psicoanalítica. Puesto que nos encontramos ante textos vivos, es decir, conectados estrechamente unos con otros, en los que se visualizan las ideas de Lacan expuestas en sus conferencias o seminarios, el estudio y discusión de teorías de filósofos, científicos y artistas, la resignificación y actualización de ideas provenientes de sus indagaciones clínicas, así como la incorporación de juegos de palabras y formulaciones matemáticas que le sirvieron para exponer los temas.

\footnotetext{
${ }^{11}$ Beillerot, «La relación con el saber: una noción en formación». Y Beillerot, «Le rapport au savoir».

12 Magalí Pastorino, «Proceso productivo: sujeto y saber en la enseñanza universitaria de artes visuales», Revista Horizontes Filosóficos, n. ${ }^{\mathrm{o}} 10$ (2020), 93-106.

${ }^{13}$ Beillerot, «La relación con el saber: una noción en formación». Y Charlot, La relación con el saber: elementos para una teoría.

${ }^{14}$ Cavalcanti, «Le rapport au savoir: émergence, propagation et institutionnalisation en tant que notion dans les domaines des Sciences de l'Éducation et des Didactiques». Y Vercellino, «La relación con el saber: revisitando los comienzos del concepto».

15 Jacques Lacan, «La subversión del sujeto y dialéctica del deseo en el inconsciente freudiano». En Escritos II, Jacques Lacan (México: Siglo Veintiuno Editores, 2018), 755-787.

16 Jacques Lacan, «La ciencia y la verdad». En Escritos II, Jacques Lacan (México: Siglo Veintiuno Editores, 2018), 813-834.

${ }^{17}$ Élisabeth Roudinesco, Lacan, frente y contra todo (Buenos Aires: Fondo de Cultura Económica, 2012).
} 


\section{विимви}

Wimblu, Rev. Estud. de Psicología UCR, 17(1) 2022 (Enero-Junio): 33-52 /ISSN: 1659-2107

En esta ocasión, y como ya se mencionó, nuestra propuesta es revisar la noción de relación con el saber en la vía de Beillerot ${ }^{18}$ para cotejarla a la luz de las obras lacanianas antedichas y actualizarla en función de las situaciones observadas en los procesos productivos de la formación universitaria en arte visual.

\section{Relación con el saber desde la perspectiva de Jacky Beillerot}

Con respecto a la noción relación con el saber propuesta por Beillerot, ${ }^{19}$ señalamos tres planos de análisis: el que refiere al estudio de la frase relación con y la revisión, análisis y discusión de las nociones de saber y sujeto tomadas de debates contemporáneos; el derivado de las diferencias con Charlot, ${ }^{20}$ acerca de los alcances de la noción y, por último, la idea de proceso creador como una característica de la relación con el saber. Este último, en particular, nos permite aproximarnos a la comprensión del proceso productivo en la formación universitaria en arte visual.

En primer lugar, para precisar la noción, Beillerot parte del estudio de la frase relación con, de las nociones de saber y de sujeto que subyacen en la articulación. ${ }^{21}$ Con respecto a los alcances de la frase relación con (rapport au), Beillerot realiza una comparación con otra semejante, relación de (rapport de), encontrando que la primera supone - y remite a - una exterioridad, es decir, indica una relación con «algo» que, en cierta medida, se halla fuera del ámbito del sujeto, pero que, a la vez, sugiere a una totalidad que abarca un sujeto y un objeto en relación. ${ }^{22}$

Por una parte, la frase tiene el poder de evocar algo exterior sin reducirlo, que es el saber entendido como una representación del stock de saberes, usos y modos sociales. Y, por otra parte, esta no representa una «tramoya cognitiva y estratégica» del sujeto, ${ }^{23}$ es decir, a la toma de distancia de un sujeto — sí mismo — para la obtención de un «dato psíquico». Por el contrario, remite a una relación íntima que permitiría comunicar el sentido común de un saber colectivo.

\footnotetext{
${ }^{18}$ Beillerot, «La relación con el saber: una noción en formación». Y Beillerot, «Le rapport au savoir».

${ }^{19}$ Beillerot, «La relación con el saber: una noción en formación». Y Beillerot, «Le rapport au savoir».

${ }^{20}$ Charlot, La relación con el saber: elementos para una teoría.

${ }^{21}$ Beillerot, «La relación con el saber: una noción en formación». Y Beillerot, «Le rapport au savoir».

${ }^{22}$ Beillerot, «La relación con el saber: una noción en formación».

${ }^{23}$ Beillerot, «Le rapport au savoir», 46.
} 
Ahora bien, desde esta perspectiva, no se tiene una relación con el saber, más bien se es en una relación en la que los actos y conductas testificarían y transcribirían el vínculo del deseo y el saber.

Es por ello que, según Beillerot, ${ }^{24}$ esta noción es útil para indagar en el proyecto de un sujeto, entendido como autor - que autoriza y autentifica - que sabe, que sabe no saber, que sabe saber sin saberlo bien, que sabe querer saber, que...; porque supone la disposición de alguien hacia el saber. Por lo tanto, corresponde a la perspectiva del sujeto y su «comercio amoroso».

En este sentido, es una «noción hablante» ${ }^{25}$ que señala una configuración sin explicitar sus términos, en la que el sujeto en su singularidad está sobreentendido; además, sugiere una causa indispensable. En efecto, dicha noción designaría el vínculo como condición, no como operación o mecanismo. ${ }^{26}$

Con respecto al saber, señala dos fuentes etimológicas de origen europeo, ${ }^{27}$ una germana, en la que saber se asocia al ver, a la imagen y a la forma - y que llevaría al discernimiento - y otra latina, relativa a la experiencia y sabiduría. No obstante, para el investigador, la centralidad y el redimensionamiento de la noción de saber en el pensamiento francés es un fenómeno contemporáneo, vinculado con la repercusión de la obra foucaultiana, ${ }^{28}$ en la que el saber (o saberes) se define formando parte de las prácticas discursivas sociales. Por lo cual, la noción pasa a distinguirse evidentemente de otras tales como ciencia y conocimiento.

Ahora bien, Beillerot señala la doble paradoja que resulta de articular la frase con la noción de saber de Foucault. La primera es que:

... la extensión del saber da lugar al reconocimiento de todos los saberes de los individuos, precisamente por parte de quienes se esfuerzan por eliminar al sujeto de la historia; segunda paradoja, la relación con el saber, que sólo puede entenderse como la del sujeto, no lo nombra, y de este modo preserva el aspecto materialista de la noción. ${ }^{29}$

A causa de ello, la inclusión del sujeto en la cuestión de la relación con el saber solo puede entenderse como la de un cierto sujeto — que no es nombrado-y el énfasis del sintagma está puesto en la relación entendida como un proceso creador. De modo que,

\footnotetext{
${ }^{24}$ Beillerot, «Le rapport au savoir».

${ }^{25}$ Beillerot, «La relación con el saber: una noción en formación».

${ }^{26}$ Beillerot, «La relación con el saber: una noción en formación».

${ }^{27}$ Beillerot, «La relación con el saber: una noción en formación».

${ }^{28}$ Michel Foucault, Arqueología del saber (Buenos Aires: Siglo Veintiuno Editores, 2010).

${ }^{29}$ Beillerot, «La relación con el saber: una noción en formación», 49.
} 
recuperar el sintagma le permite tomar distancia del efecto de sustanciación del sujeto y/o del objeto.

Y, por ello, señala que Lacan, «sensible al estructuralismo», fue obligado a reintroducir el sujeto, «cierto sujeto, en especial utilizando la noción de relación con el saber, de la cual elimina el yo [je]», ${ }^{30}$ pero también echar luz sobre la participación e inscripción del saber en la historia psíquica y social del sujeto, en el horizonte fantasmático de un saber absoluto. ${ }^{31}$

La inclusión de cierto sujeto en la relación con el saber tiene varios efectos. Primero, revela la matriz filosófica moderna que amolda las nociones de saber y conocimiento. ${ }^{32}$ Segundo, se trata de un saber indisociable de la acción, de su puesta en práctica, del acto. Asimismo, es un saber hablado emergente de condiciones socioculturales, que se ejerce con otros, por tanto, es una práctica social de saberes que supone una conciencia de saber. Tercero, involucra la dimensión del deseo, de la concepción de sujeto de la teoría psicoanalítica. ${ }^{33}$

Sin ninguna duda, fue Freud, ${ }^{34}$ en «Tres ensayos de teoría sexual» publicado en 1905, el primero en afirmar, basado en la clínica, que el deseo de saber no es natural, en contraposición a la tesis aristotélica que postula que la pasión por conocer es connatural en el hombre. Esta pulsión específica del deseo de saber emerge en la vida infantil apuntalada por otras pulsiones primigenias, y, si bien implica la singularidad del sujeto, no se sustrae del influjo de la cultura.

La falta, que es la condición constitutiva del sujeto deseante, es necesaria para cualquier proceso que involucre el deseo. Y como señala Venturini, la angustia

... no es propiamente la falta, la ausencia misma no es la causante de la angustia. Lo que angustia es la falta de esta falta [...] es cuando no hay espacio para que se cumpla la función

\footnotetext{
${ }^{30}$ Beillerot, «La relación con el saber: una noción en formación», 49.

31 «Tout savoir individuel est donc partiel et s'inscrit dans I 'histoire psychique et sociale du sujet sur I 'horizon fantasmatique d'un savoir absolu», Beillerot, «Le rapport au savoir», 17.

32 «Approfondir les distinctions entre savoir et connaissance est une attitude pertinente, d'autant que les deux concepts ont une longue histoire philosophique, mais aussi une histoire de leurs usages sociaux; que le savoir soit décrété ce qui est acquis par transmission, et la connaissance comme la découverte par soimême de ce qui est juste et vrai, c'est-à-dire l'exercice de la vérité et de la vérification, pennet sans doute de mieux comprendre le mouvement psychologique et social du lien entre les deux concepts», Beillerot, «Le rapport au savoir», 24.

${ }^{33}$ Beillerot, «La relación con el saber: una noción en formación».

${ }^{34}$ Sigmund Freud, «Tres ensayos para una teoría sexual». En Los textos fundamentales del psicoanálisis, Sigmund Freud (Barcelona: Altaya, 1993).
} 


\section{विимви}

Wimblu, Rev. Estud. de Psicología UCR, 17(1) 2022 (Enero-Junio): 33-52 /ISSN: 1659-2107

del deseo, cuando no hay lugar para que el significante pueda significar. La falta es lo que produce deseo, al faltar la falta, el sujeto es perturbado, ya no tiene lugar para desear. ${ }^{35}$

Por lo dicho, si consideramos que la producción artística supone un proceso productivo, no podemos descuidar el lugar de la falta y del deseo y sus efectos en el obrar artístico.

Ahora bien, dada la proximidad del saber con el acto, la relación con el saber implicaría un proceso en el que se producen transformaciones, pero no en el sentido de la dialéctica hegeliana; es un proceso creador que en cierta medida involucra un saber sobre «sí»y lo «real» basado en el juego de los saberes disponibles de la época, como veremos más adelante.

En lo que refiere al segundo plano de análisis, las diferencias de Beillerot con Charlot marcan su distancia de una fenomenología. Pues, para Charlot, ${ }^{36}$ de la relación con el saber emerge un sentido, el cual es «producido por una puesta en relación, en el interior de un sistema o en las relaciones con el mundo o con los otros». Beillerot acota que el sentido involucra la autonomía y la libertad del individuo frente a las cuestiones fundamentales humanas, colocando el énfasis en lo cognitivo y restringiendo los alcances del sintagma al campo de la conciencia. ${ }^{37}$ Sostiene que el ámbito de la conciencia no cubre la condición singular del sujeto en cuanto «historia no historizada del dédalo de la construcción psíquica», ${ }^{38}$ con sus emociones, represiones, angustias y aspiraciones personales.

También discrepa en definir la noción relación con el saber como una forma específica de la relación con el aprender. ${ }^{39}$ Por el contrario, para Beillerot aprender implica específicamente aceptar las limitaciones intrínsecas de las cosas a aprender, ${ }^{40} \mathrm{es}$ decir, someterse al dogma, e involucra la afiliación del sujeto. En pocas palabras, aprender

\footnotetext{
35 Joaquín Venturini, «La angustia en la enseñanza: entre lo inesperado y la falta de la falta». En Evocar la falta: la angustia y el deseo del enseñante, Ana María Fernández Caraballo y Raumar Rodríguez Giménez (Montevideo: Psicolibros-Waslala, 2011), 62.

${ }^{36}$ Charlot, La relación con el saber: elementos para una teoría, 65.

${ }^{37}$ Beillerot, «Le rapport au savoir».

38 «Histoire non historicisée du dédale de la construction psychique dont des étapes ou des stades sont nommés par Freud et ses successeurs, mais comme autant de catégories "plaquées" sur un vécu, c'est-à-dire des émotions, des refoulements, des angoisses et des aspirations», Beillerot, «Le rapport au savoir», 43.

${ }^{39}$ Charlot, La relación con el saber: elementos para una teoría.

40 «Apprendre demeure une activité plus ou moins intentionnelle, comme nous l'avons dit, qui implique de la part de chaque sujet une soumission; phénomène essentiel qui pennet de comprendre que le refus d'apprendre, lui aussi plus ou moins intentionnel, est un refus de soumission. Pour apprendre, en effet, le sujet accepte, de fait, de ne pas savoir, puis accepte avec plus ou moins de facilité, les contraintes intrinsèques des choses à apprendre», Beillerot, «Le rapport au savoir», 44.
} 
es admitir las respuestas de los otros, mientras que la relación con el saber es un proceso creador.

A pesar de sus diferencias, ambos investigadores señalan que la noción relación con el saber tiene origen en un sintagma de la obra lacaniana de los años sesenta. ${ }^{41}$

Ahora bien, Beillerot señala el gran desarrollo que tuvo el sintagma lacaniano al ser retomado por el pensamiento francés en la segunda mitad del siglo pasado, de parte de psicoanalistas y marxistas, así como también, por su aplicación en el campo de la educación, en concreto, en el campo de la capacitación de adultos. En este derrotero, la noción se ubicó «en el centro de los debates más intensos y originales de los últimos veinte años [por lo que] la expresión es propia de su época». ${ }^{42}$ El contexto de dichos debates forma parte del panorama del pensamiento francés, por ende, el sintagma se complejizó y se densificó al integrar la discusión acerca del sujeto y del saber desde diversas corrientes. A este respecto, Badiou señala la pugna de dos corrientes filosóficas francesas de pensamiento, originadas a comienzos del siglo pasado, una centrada en la vida y otra en el concepto, las cuales en el correr de la segunda mitad del siglo pasado condujeron a la problematización de la noción cartesiana de sujeto. En tal escenario, el psicoanálisis tuvo el rol de interlocutor de la filosofía, pues «la gran invención freudiana fue precisamente una nueva proposición acerca del sujeto»; ${ }^{43}$ porque en cierta medida «el inconsciente de Freud [...] es también algo simultáneamente vital y simbólico, portador del concepto». ${ }^{44}$

Con respecto al tercer plano de análisis, la relación con el saber entraña un proceso creador que es singular y único, no es reproducible ni transmitible; por el contrario, el «dar cuenta» o «darse cuenta» es una formación de análisis que surge del estar en «lo sabido» en la que algo siempre se pierde, por lo que «es íntegramente la relación del proceso creador de saber con lo sabido y no solo lo sabido, de lo que se obtendrían respuestas o soluciones». ${ }^{45}$

En consecuencia, la creación de saber por una parte es contraria a «una racionalidad demasiado unívoca». ${ }^{46}$ Por la otra, no es un mero proceso individual, porque

41 Vercellino, «Revisión bibliográfica sobre la relación con el saber: desplazamientos teóricos y posibilidades para el análisis psicopedagógico de los procesos escolares».

42 Beillerot, «La relación con el saber: una noción en formación», 51.

${ }^{43}$ Alain Badiou, La aventura de la filosofia francesa (Buenos Aires: Eterna Cadencia, 2013), 20.

${ }^{44}$ Badiou, La aventura de la filosofia francesa, 21.

${ }^{45}$ Beillerot, «La relación con el saber: una noción en formación», 67.

${ }^{46}$ Beillerot, «La relación con el saber: una noción en formación», 72. 
el acto humano no está aislado, implica la cultura histórica — los códigos y modos de obrar-y se desarrolla en una organización social.

En lo que refiere a nuestros propósitos, se evidencia que el obrar artístico es el paradigma del proceso creador. Dicho obrar tiene como característica la producción, ${ }^{47}$ es decir, la capacidad de hacer presente un ser que no estaba antes del obrar, es el territorio más apto para revisar la relación con el saber si consideramos el saber del arte no como la técnica aplicada para la creación, sino como la subjetivación de naturaleza inconsciente en su singularidad, que involucra un estado de incertidumbre e indeterminación, y que se encuentra adherida a las formaciones analíticas.

\section{El sintagma lacaniano en «La subversión del sujeto y dialéctica del sujeto en el inconsciente freudiano»}

Como mencionáramos al comienzo, relación con el saber es un sintagma que introduce Lacan en su obra de los años sesenta, el cual le permite postular un sujeto del significante y sugerir una subjetivación por el significante. ${ }^{48}$

Brevemente, en «La subversión del sujeto y dialéctica del sujeto en el inconsciente freudiano» se presenta el drama del ser visto desde la clínica psicoanalítica con relación a la noción de sujeto provisto por la filosofía moderna — sujeto absoluto hegeliano- $\mathrm{y}$ por la ciencia — sujeto abolido-.

En este marco, el psicoanálisis representa un sismo para la ciencia moderna al sobrevenir en ella y anudar más íntimamente el régimen de saber con el de verdad, ${ }^{49}$ que quedaron separados a partir del sujeto inaugurado por Descartes. A este respecto, para Lacan, el cogito se encuentra en el núcleo del espejismo que constituye al hombre moderno, en la búsqueda de certezas para poder actuar correctamente.

De esta manera, la subversión del sujeto realizada por el psicoanálisis es el movimiento por el que se revela el sujetamiento del ser a la metafísica moderna del hombre. $^{50}$

\footnotetext{
${ }^{47}$ Giorgio Agamben, El hombre sin contenido (Barcelona: Altera, 2005).

${ }^{48}$ Lacan, «La subversión del sujeto y dialéctica del deseo en el inconsciente freudiano», 770.

${ }^{49}$ Lacan, «La subversión del sujeto y dialéctica del deseo en el inconsciente freudiano», 759.

${ }^{50}$ Ana Ruth Najles, «Teoría del sujeto». En Jacques Lacan: el psicoanálisis y su aporte a la cultura contemporánea, editado por Miriam Chorne y Gustavo Dessal (Madrid: Fondo de Cultura Económica, 2017), 137-142.
} 


\section{arin}

Wimblu, Rev. Estud. de Psicología UCR, 17(1) 2022 (Enero-Junio): 33-52 /ISSN: 1659-2107

Lacan didácticamente toma de la fenomenología hegeliana el sintagma para definir al sujeto, a partir de una mediación, en vez de concebirlo en las condiciones de su emergencia; esta estrategia le permite revisar el postulado metafísico que funda al sujeto moderno. Así, el sintagma aparece asociado a la presentación del esquema hegeliano de la fenomenología del espíritu que, según Lacan, tiene la virtud de presentar una mediación fácil para situar al sujeto en una relación con el saber. ${ }^{51}$

De esta forma, muestra la ambigüedad que entraña tal relación en el ámbito de la ciencia: el sujeto —el científico- desconoce los efectos de tal sujeción. Justamente, el desconocimiento es la condición del sujeto de la ciencia. ${ }^{52}$

A diferencia de la psicología que postula la unidad del sujeto y, en correlato, un conocimiento que se supone connatural — pero que no lleva a otra cosa que «a perpetuar un marco académico $»^{53}$ _, el psicoanálisis indaga en la «frontera sensible de la verdad y del saber» que es aquello que la ciencia «parece ciertamente haber regresado a la solución de cerrarla». ${ }^{54}$

Para comprender el sintagma, consideramos que es necesario seguir el modo en que Lacan expone la diferencia entre el postulado del sujeto moderno y el del sujeto del psicoanálisis, en el que identificamos tres planos articulados: el de la relación del sujeto con la verdad, el del sujeto del significante y el de la relación del sujeto y el deseo. A continuación, presentamos brevemente las ideas principales de cada plano.

En lo que refiere al plano de la relación del sujeto con la verdad, Lacan toma el esquema de la fenomenología hegeliana para demostrar el error que se constituye respecto a la verdad, que «no es otra cosa sino aquello de lo cual el saber no puede enterarse de que lo sabe sino haciendo actuar su ignorancia». ${ }^{55}$ Porque en dicho esquema la verdad dialécticamente converge en el saber absoluto, generando una solución ideal a la vez que el efecto perturbador de la verdad se reabsorbe constantemente en su dinámica. En consecuencia, la verdad se reduce a la

... conjunción de lo simbólico con un real del que ya no hay nada que esperar, ¿qué es esto sino un sujeto acabado en su identidad consigo mismo? En lo cual se lee que ese sujeto está ya perfecto allí y que es la hipótesis fundamental de todo este proceso. ${ }^{56}$

\footnotetext{
${ }^{51}$ Lacan, «La subversión del sujeto y dialéctica del deseo en el inconsciente freudiano», 755.

${ }^{52}$ Lacan, «La subversión del sujeto y dialéctica del deseo en el inconsciente freudiano», 756.

${ }^{53}$ Lacan, «La subversión del sujeto y dialéctica del deseo en el inconsciente freudiano», 756.

${ }^{54}$ Lacan, «La subversión del sujeto y dialéctica del deseo en el inconsciente freudiano», 759.

${ }^{55}$ Lacan, «La subversión del sujeto y dialéctica del deseo en el inconsciente freudiano», 759.

${ }^{56}$ Lacan, «La subversión del sujeto y dialéctica del deseo en el inconsciente freudiano», 759.
} 
En este esquema, el sujeto directamente designa el sustrato, Selbstbewusstsein, omnisciente. Entonces, a partir de señalar el error del sujeto abolido de la ciencia y del sujeto absoluto hegeliano, que convergen en la concepción del sujeto moderno, Lacan marca el sentido del programa de Freud que es el «regreso de la verdad al campo de la ciencia, con el mismo movimiento con que se impone en el campo de su praxis: reprimida, retorna allí». ${ }^{57}$

La verdad es lo proferido, las palabras dichas que interrumpen el discurso efectivo del hablante: «el inconsciente, a partir de Freud, es una cadena de significantes que en algún sitio [...] se repite e insiste para interferir en los cortes que le ofrece el discurso efectivo y la cogitación que él informa». 58

En el plano de la noción de sujeto del significante, Lacan une la teoría del sujeto con la del significante, defendiendo la concepción de un sujeto sin sustancia, en la que el yo es simplemente un objeto que cumple con una función imaginaria de unidad. Y, en este sentido, el sujeto del psicoanálisis evidencia la captura del viviente en las redes del significante. $^{59}$

Por ello, «un significante es lo que representa al sujeto para otro significante», ${ }^{60}$ es decir, que dicha captura del ser en la sujeción al significante implica al menos dos significantes para que el sujeto emerja. Esto significa, por un lado, que el significante por sí mismo no significa, solo vale con relación a otro, aunque tampoco así consigue la designación absoluta. Y, por el otro, que el funcionamiento de la cadena de significantes da lugar a un sujeto concebido como intervalo, escansión y ruptura. En efecto, el sujeto no muestra ninguna consistencia que lo singularice ni haga equivaler a los significantes, pues no lo representan. En todo caso, es el tercero (el inconsciente) que le da acceso a un régimen identitario que el sujeto, como tal, no posee. ${ }^{61}$

El corte que produce la palabra correspondiente a la verdad en el discurso efectivo muestra que la estructura del sujeto es discontinua con respecto a lo real, ${ }^{62}$ esto es la causa del hablante.

\footnotetext{
${ }^{57}$ Lacan, «La subversión del sujeto y dialéctica del deseo en el inconsciente freudiano», 760.

${ }^{58}$ Lacan, «La subversión del sujeto y dialéctica del deseo en el inconsciente freudiano», 760.

${ }^{59}$ Najles, «Teoría del sujeto».

${ }^{60}$ Lacan, «La subversión del sujeto y dialéctica del deseo en el inconsciente freudiano», 779.

${ }^{61}$ Guy Le Gaufey, El sujeto según Lacan (Buenos Aires: El Cuenco del Plata, 2016).

${ }^{62}$ Lacan, «La subversión del sujeto y dialéctica del deseo en el inconsciente freudiano», 762.
} 


\section{विимви}

Wimblu, Rev. Estud. de Psicología UCR, 17(1) 2022 (Enero-Junio): 33-52 /ISSN: 1659-2107

En consecuencia, el sujeto del inconsciente se presenta en actos inesperados y desconocidos para la unidad yoica consciente. Es el sujeto dividido por efecto del significante, lanzado a buscar el significante faltante:

... este sujeto no es más que el pliegue significante modelado en la actualidad de un cuerpo de carne y de lengua al que lleva por el hecho de la pura potencia que es la suya en tanto sujeto, hacia el cumplimiento de sus potencialidades. ${ }^{63}$

Es así que el inconsciente se estructura como lenguaje, con una dinámica que efectúa por sustitución y combinación del significante, metáfora y metonimia, como lo reconoció Freud en los procesos primarios a nivel sincrónico y diacrónico del discurso.

Por ello, en este marco, el yo es el shifter o indicativo del sujeto en cuanto acto de habla, «designa el sujeto de la enunciación, pero no lo significa», ${ }^{64}$ no sabe lo que dice ni que habla.

Por lo tanto, el inconsciente es un saber sin sujeto, un saber que no requiere de un ser consciente que lo sepa, y el sujeto es afectado por no saber.

El sujeto que habla aquí se encuentra, pues, desposeído de sí mismo y su experiencia se revela como inherentemente fragmentaria, carente de identidad, motivo por el cual busca, pide, demanda, decíamos, el significado. ${ }^{65}$

En el plano de la relación del sujeto y el deseo, Lacan parte críticamente de los postulados hegelianos. Señala el error de realizar una reducción logizante por el cual el sujeto absoluto siempre sabe lo que quiere y la verdad es inmanente a la realización del saber. Al contrario de Freud, que reúne el saber y la verdad, la verdad como palabra y estructura de ficción, ${ }^{66}$ en el cual el deseo se anuda al deseo del Otro, en cuyo lazo puede residir el deseo de saber. ${ }^{67}$

En este sentido, el lenguaje entrelaza la condición prematura del sujeto con el deseo y el saber. Y, de esta manera, el yo se constituye por el camino de la subjetivación del significante. ${ }^{68}$

\footnotetext{
${ }^{63}$ Le Gaufey, El sujeto según Lacan, 133.

${ }^{64}$ Lacan, «La subversión del sujeto y dialéctica del deseo en el inconsciente freudiano», 761.

65 Santiago Cardozo González, La necesidad del significante: una crítica a la razón referencial (Montevideo: CSIC Biblioteca Plural, 2021), 107.

${ }^{66}$ Lacan, «La subversión del sujeto y dialéctica del deseo en el inconsciente freudiano», 768.

${ }^{67}$ Lacan, «La subversión del sujeto y dialéctica del deseo en el inconsciente freudiano», 764.

${ }^{68}$ Lacan, «La subversión del sujeto y dialéctica del deseo en el inconsciente freudiano», 770.
} 


\section{विимви}

Wimblu, Rev. Estud. de Psicología UCR, 17(1) 2022 (Enero-Junio): 33-52 /ISSN: 1659-2107

Así, el deseo parte de la estructura del significante ${ }^{69}$ como un saber inscripto en un discurso que se desconoce y se desliza en la cadena significante como la significación. Por lo tanto, el sujeto está fijado a un modo de satisfacción pulsional.

Así arribamos a la relación del deseo y el sujeto del significante en la historia de la constitución del sujeto. En breves palabras, el deseo se define en su irreductibilidad a la demanda y a la necesidad: «no hay demanda que no pase de una manera o de otra por los desfiladeros del significante» ${ }^{70}$ incluso se encuentra en el «margen donde la demanda se desgarra de la necesidad», en el llamado dirigido al Otro que al no tener satisfacción universal se constituye en una falla y el advenimiento de la angustia. ${ }^{71}$

Por ello, el deseo no está solamente sometido a los accidentes de la historia del sujeto, sino que es el concurso de elementos estructurales, ya que «el deseo del hombre es el deseo del Otro [...] en cuanto Otro como desea». ${ }^{72}$ No es natural: su «artificio [es] gramatical». ${ }^{73}$

Dicha historia del sujeto es presentada a través del grafo del deseo, que explica la situación del deseo con relación a un sujeto definido a través de la articulación con el significante, en donde el Otro tiene un rol importante por ser la sede del sujeto del significante..$^{74} \mathrm{Y}$, en el juego de las pulsiones, el organismo se actualiza con el Otro y encuentra la dimensión del significante que lo propulsa en su destino de sujeto.

Así, «el Otro como sede del puro sujeto del significante ocupa allí la posición maestra, incluso antes de venir allí a la existencia [...] no se puede ni siquiera hablar de código si no es ya el código del Otro», ${ }^{75}$ en el mensaje se constituye el sujeto, por lo tanto, lo que puede emitir es del Otro, pero, además, no concibe un metalenguaje, no hay Otro del Otro. ${ }^{76}$

De este modo, se observa la condición del sujeto del significante, configurado en la dinámica de la castración, el goce, el placer y la ley, ${ }^{77}$ que no desarrollaremos en esta oportunidad.

\footnotetext{
${ }^{69}$ Lacan, «La subversión del sujeto y dialéctica del deseo en el inconsciente freudiano», 764.

${ }^{70}$ Lacan, «La subversión del sujeto y dialéctica del deseo en el inconsciente freudiano», 772.

${ }^{71}$ Lacan, «La subversión del sujeto y dialéctica del deseo en el inconsciente freudiano», 774.

${ }^{72}$ Lacan, «La subversión del sujeto y dialéctica del deseo en el inconsciente freudiano», 775.

${ }^{73}$ Lacan, «La subversión del sujeto y dialéctica del deseo en el inconsciente freudiano», 777.

${ }^{74}$ Lacan, «La subversión del sujeto y dialéctica del deseo en el inconsciente freudiano», 767,773.

${ }^{75}$ Lacan, «La subversión del sujeto y dialéctica del deseo en el inconsciente freudiano», 767.

${ }^{76}$ Lacan, «La subversión del sujeto y dialéctica del deseo en el inconsciente freudiano», 773.

${ }^{77}$ Lacan, «La subversión del sujeto y dialéctica del deseo en el inconsciente freudiano», 779-782.
} 
Por lo dicho, el sujeto del significante es el soporte de una existencia singular:

Es necesario, pues, contentarse con adjuntar esta entidad del sujeto del significante a la monótona cualidad del «existir», en adelante entendida no como un don divino, sino como puro enganche al campo del Otro, en tanto tesoro de los significantes, por el hecho de la puesta en juego de una palabra, que de manera tan rudimentaria como se quiera, moviliza el armazón del lenguaje en el cual el humano se encuentra tomado desde sus primeros pasos. ${ }^{78}$

\section{El sintagma en «La ciencia y la verdad»}

El sintagma en esta obra nos lleva al cuestionamiento del sujeto moderno, a la operación generalizante de la epistemología que pretende incluir el acontecimiento en un conjunto constante y calculable, y que lleva a que el sujeto sea «un resto de la confrontación del orden simbólico con el ser viviente». ${ }^{79}$ De allí que Lacan coloque al psicoanálisis formando parte de las ciencias conjeturales, es decir, aquellas que parten del lenguaje como ley instituyente de la cultura y que, además, a la vez que precede al sujeto, lo constituye.

El punto crucial de su crítica es el cogito cartesiano. Lacan afirma que es revolucionario porque funda un sujeto cuya existencia es puntual, que solo puede dar cuenta de su existencia si se encuentra pensando.

En procura de estar en estado puro, todos los atributos del ser - experiencia, ideas, representaciones - caen vaciándose de subjetividad y la verdad es dejada a Dios. Porque lo que le importa es seguir su método y ocuparse de la producción de un saber útil para la acción, un saber universal, desubjetivado, válido para todos los sujetos.

En este sentido, Lacan señala que el sujeto cartesiano es un «cierto momento del sujeto» que en procura de sujetar el ser al cogito se convierte en el «desfiladero de un rechazo de todo saber». ${ }^{80}$

Dicho sujeto, que también llamará sujeto de la ciencia, «debe tomarse en el sentido de puerta estrecha» y surge con la «división experimentada del sujeto como división entre el saber y la verdad», como se mencionó antes. ${ }^{81}$

Ahora bien, Lacan advierte que la praxis psicoanalítica no se puede comprender sin el cientificismo que se desarrolla a partir del sujeto de la ciencia, porque está

\footnotetext{
${ }^{78}$ Le Gaufey, El sujeto según Lacan, 134.

79 Esteban Laso, «Psicoanálisis y epistemología». En La posciencia: el conocimiento científico en las postrimerías de la modernidad, editado por Esther Díaz (Buenos Aires: Biblos, 2000), 325.

${ }^{80}$ Lacan, «La ciencia y la verdad», 814.

${ }^{81}$ Lacan, «La ciencia y la verdad», 814.
} 
implicado en la matriz moderna. Lo prueba el momento de ruptura entre Freud y Jung, este último postuló un sujeto dotado de profundidad, irreductible a la ciencia moderna «la cual no es nada más que la relación que definimos [...] como puntual y desvaneciente, esa relación con el saber que de su momento históricamente inaugural ha conservado el nombre de cogito». ${ }^{82}$

En efecto, relación con el saber inaugura el sujeto cartesiano que supone la experimentación de la verdad y el saber de manera separada. Y es en este sujeto sobre el que opera el psicoanálisis. ${ }^{83}$ Por consiguiente, el sujeto del psicoanálisis incorpora el sujeto cartesiano, que, a la vez que existe solo en cuanto piensa, excluye las formaciones inconscientes.

En el recorrido de distinguir la ciencia y el psicoanálisis, afirma, primero, que no existe la ciencia del hombre, solo su sujeto. ${ }^{84} \mathrm{Y}$, segundo, que el objeto del psicoanálisis, que llama objeto a (objeto a minúscula), no define una relación epistemológica, «puesto que ese objeto a debe insertarse, ya lo sabemos, en la división del sujeto por donde se estructura muy especialmente», ${ }^{85} \mathrm{y}$, además, «este objeto $a$ no está tranquilo», ${ }^{86}$ es decir, que no es claro ni discreto.

En este contexto, Lacan modifica la consigna freudiana introduciendo la cuestión del sujeto y corriendo de lugar la cuestión del yo: Wo Es war, soll Ich werden, «allí donde ello era, allí como sujeto debo advenir yo». ${ }^{87}$

Dicha modificación le permite exponer la cuestión de la causa del ser, que no será tomada como categoría lógica, sino «causando todo el efecto», es decir, la «verdad como causa y el saber puesto en ejercicio», ${ }^{88}$ al contrario de la fórmula cartesiana que coloca la causa en el cogito. No obstante, en un juego inspirado en la crítica heideggeriana y en la inclinación de Lacan por la oralidad sobre lo escrito, plantea que: «pienso, "luego soy", con comillas alrededor de la segunda cláusula, se lee que el pensamiento no funda el ser, sino anudándose en la palabra donde toda operación toca a la esencia del lenguaje». ${ }^{89}$

\footnotetext{
82 Lacan, «La ciencia y la verdad», 815.

${ }^{83}$ Lacan, «La ciencia y la verdad», 816.

${ }^{84}$ Lacan, «La ciencia y la verdad», 817.

${ }^{85}$ Lacan, «La ciencia y la verdad», 820.

${ }^{86}$ Lacan, «La ciencia y la verdad», 821.

${ }^{87}$ Lacan, «La ciencia y la verdad», 821.

${ }^{88}$ Lacan, «La ciencia y la verdad», 825.

${ }^{89}$ Lacan, «La ciencia y la verdad», 821.
} 
Es entonces que podemos encontrar el lugar de la verdad en la trama del sujeto del psicoanálisis en la palabra dicha, no en lo que ella entraña o de lo que de ella se puede interpretar, pues como mencionamos antes «no hay metalenguaje [...] puesto que la verdad se funda por el hecho de que habla, y puesto que no tiene otro medio para hacerlo». ${ }^{90}$

No es cualquier palabra, está vinculada con el inconsciente, pues «piensen en la cosa innombrable que, de poder pronunciar estas palabras, iría al ser del lenguaje, para escucharlas como deben ser pronunciadas, en el horror [...] pero en esta revelación cada uno pone lo que puede poner». ${ }^{91} \mathrm{Y}$, «una verdad que habla tiene poco en común con un noúmeno que, tan lejos como pueda recordar la razón pura, cierra la boca». ${ }^{92}$

En este sentido, la conciencia postulada por la filosofía moderna — secuela histórica del cogito cartesiano- es «la acentuación engañosa de la transparencia del Yo [je] en acto a expensas de la opacidad del significante que lo determina». ${ }^{93}$

\section{Conclusiones}

En este derrotero, podemos observar que la definición de relación con el saber de Beillerot involucra el proceso creador y se distingue del fenómeno del aprender. Esta distinción hace que sea más pertinente a las situaciones observadas emergentes de los procesos productivos de estudiantes avanzados en arte visual, quienes se embarcan, en principio, en la experimentación de los recursos plásticos y visuales, y que cuentan con asesoramiento y asistencia técnica.

A la luz de la teoría psicoanalítica expuesta hemos profundizado en algunos aspectos de dicha noción.

En primer lugar, se trata de la relación con el saber de un cierto sujeto, el cual emerge en un régimen de saber y verdad escindidos, esto es, sujeto a un modo de satisfacción pulsional en el que está implicado el lenguaje y el Otro en la dinámica del deseo, en el juego de la falta y la angustia.

\footnotetext{
${ }^{90}$ Lacan, «La ciencia y la verdad», 824.

${ }^{91}$ Lacan, «La ciencia y la verdad», 823.

92 Lacan, «La ciencia y la verdad», 825.

${ }^{93}$ Lacan, «La subversión del sujeto y dialéctica del deseo en el inconsciente freudiano», 770.
} 
En este sentido, las situaciones observadas inmotivadas corresponden a dicho régimen del no saber del yo y del saber del sujeto del inconsciente y configura el proceso creador.

De este modo, se pueden considerar como parte necesaria de los procesos productivos en arte, que al no ser conscientes no correspondería explorarlos con herramientas que apuntan a la producción de formaciones analíticas, en este sentido, optamos por una lectura de los insumos producidos en los seguimientos en clave discursiva. Y, en el escenario de la enseñanza artística, ello supone dar lugar a situaciones observadas e integrarlas como parte del proceso creador.

En segundo lugar, las consideraciones epistemológicas lacanianas, que nos muestran los efectos de la filosofía cartesiana y de la ciencia en la concepción de sujeto y de saber, nos llevan a preguntarnos por la existencia de un saber del arte excluido de la visibilidad que produce la matriz epistemológica moderna respecto a la relación sujetoobjeto, que en arte se corresponde a la relación del artista y la obra - como producto acabado - y a la técnica como mediación que lleva a la realización de la obra.

A este respecto, la noción que postulamos, el saber del arte, valdría como saber del sujeto del psicoanálisis, con relación a la verdad, el lenguaje y el Otro, en el proceso creador, o sea, como saber en acto de un cierto sujeto. Esto significa que la exploración de las situaciones observadas requiere de un abordaje que permita integrar la dimensión del deseo y del desconocimiento propio del yo. 


\section{Referencias bibliográficas}

Agamben, Giorgio. El hombre sin contenido. Barcelona: Altera, 2005.

Badiou, Alain. La aventura de la filosofía francesa. Buenos Aires: Eterna Cadencia, 2013.

Beillerot, Jacky. «La relación con el saber: una noción en formación». En Saber y relación con el saber, editado por Jacky Beillerot, Claudine Blanchard-Laville y Nicole Mosconi, 43-78. Barcelona: Paidós, 1998.

Beillerot, Jacky. «Le rapport au savoir». En Formes et formations du rapport au savoir, editado por Jacky Beillerot, Claudine Blanchard-Laville y Nicole Mosconi, 3958. Paris: L'Harmattan, 2000.

Cardozo González, Santiago. La necesidad del significante: una crítica a la razón referencial. Montevideo: CSIC Biblioteca Plural, 2021.

Charlot, Bernard. La relación con el saber: elementos para una teoría. Montevideo: Trilce, 2006.

Cavalcanti, José. «Le rapport au savoir: émergence, propagation et institutionnalisation en tant que notion dans les domaines des Sciences de l'Éducation et des Didactiques», Academia, n. ${ }^{\circ} 19$ (2020), 27-52.

Foucault, Michel. Arqueología del saber. Buenos Aires: Siglo Veintiuno Editores, 2010.

Freud, Sigmund. «Tres ensayos para una teoría sexual». En Los textos fundamentales del psicoanálisis, Sigmund Freud. Barcelona: Altaya, [1905] 1993.

Lacan, Jacques. «La subversión del sujeto y dialéctica del deseo en el inconsciente freudiano». En Escritos II, Jacques Lacan, 755-787. México: Siglo Veintiuno Editores, [1960] 2018.

Lacan, Jacques. «La ciencia y la verdad». En Escritos II, Jacques Lacan, 813-834. México: Siglo Veintiuno Editores, [1965-1966] 2018.

Laso, Esteban. «Psicoanálisis y epistemología». En La posciencia: el conocimiento científico en las postrimerías de la modernidad, editado por Esther Díaz, 303-328. Buenos Aires: Biblos, 2000.

Le Gaufey, Guy. El sujeto según Lacan. Buenos Aires: El Cuenco del Plata, 2016.

Najles, Ana Ruth. «Teoría del sujeto». En Jacques Lacan: el psicoanálisis y su aporte a la cultura contemporánea, editado por Miriam Chorne y Gustavo Dessal, 137142. Madrid: Fondo de Cultura Económica, 2017.

Pastorino, M. «Proceso productivo: sujeto y saber en la enseñanza universitaria de artes visuales». Revista Horizontes Filosóficos, n. ${ }^{\circ} 10$ (2020), 93-106. 


\section{बिимви}

Wimblu, Rev. Estud. de Psicología UCR, 17(1) 2022 (Enero-Junio): 33-52 /ISSN: 1659-2107

Roudinesco, Élisabeth. Lacan, frente y contra todo. Buenos Aires: Fondo de Cultura Económica, 2012.

Venturini, Joaquín. «La angustia en la enseñanza: entre lo inesperado y la falta de la falta». En Evocar la falta: la angustia y el deseo del enseñante, Ana María Fernández Caraballo y Raumar Rodríguez Giménez, 51-74. Montevideo: PsicolibrosWaslala, 2011.

Vercellino, Soledad. «Revisión bibliográfica sobre la relación con el saber: desplazamientos teóricos y posibilidades para el análisis psicopedagógico de los procesos escolares». Revista Electrónica Educare 19, n. ${ }^{\circ} 2$ (2015), 53-82.

Vercellino, Soledad. «La relación con el saber: revisitando los comienzos del concepto». En Aprendizajes: la escuela y los (des)encuentros con el saber, compilado por Soledad Vercellino, 41-48. Buenos Aires: Universidad Nacional de Río Negro, 2018 .

Esta obra está bajo una licencia de Creative Commons 